\title{
Radiation Dose Distribution for Patients Undergoing Routine Radiological Scans for Kidney Stone Diagnosis at the University Hospital of the West Indies \\ DC Walker ${ }^{1}$, WD Aiken ${ }^{2}, S_{\text {Shah }}^{3}$, MK Voutchkov ${ }^{1}$, L-GM Burnett ${ }^{4}$, A Gordon ${ }^{1}$, CA McKenzie
}

\begin{abstract}
Objectives: To investigate the radiation dose received by patients undergoing routine plain $\mathrm{x}$-ray and computed tomography scans for kidney stone studies.

Methods: For x-ray scans two sets of experiments were performed. Thermo-Luminescence Dosimeters were used to determine the distribution of radiation dose across the abdominal surface and an acrylic water phantom equipped with a Farmer ionization chamber was used to measure the radiation dose as X-ray photons travel through the abdomen. For CT scans, the Dose Length Product (DLP) over 3 months for abdominal studies was recorded. Calculations were done to convert the DLP to effective dose using $\mathrm{k}$ factor values from the International Commission on Radiological Protection (ICRP) report 103. The results were then mapped and analysed using Microsoft excel and Wolfram Mathematical software.
\end{abstract}

Results: Skin dose was greatest at the centre of the abdomen. Radiation equivalent dose to the skin for a plain abdominal x-ray ranged from $2.38 \mathrm{mSv}$ for patients with a sthenic body habitus to $5.45 \mathrm{mSv}$ for hypersthenic patients. For CT scans, effective dose was found to range between $6.72 \mathrm{mSv}$ and $24.27 \mathrm{mSv}$.

Conclusion: All radiation dose measured were found to be within recommended ICRP operational limits. The developed procedure could be implemented in other radiology departments in Jamaica as a part of their quality assurance program.

Keywords: Computed tomography, kidney stone, radiation dose, $\mathrm{x}$-ray scan

From: ${ }^{1}$ Department of Physics, Faculty of Science and Technology, ${ }^{2}$ Section of Urology, Department of Surgery, UWI, Faculty of Medical Sciences, ${ }^{3}$ Section of Radiology, Department of Surgery, ${ }^{4} \mathrm{X}$-Ray Department, University Hospital of the West Indies Mona, and ${ }^{5}$ School of Medical Radiation Technology, Faculty of Medical Science, The University of the West Indies Mona, Kingston, Jamaica.

Correspondence: Mr D Walker, Department of Physics, Faculty of Science and Technology, The University of the West Indies, Mona. Kingston, Jamaica.

Email: darrion.walker02@uwimona.edu.jm 


\section{INTRODUCTION}

Kidney Stones are developed from the urine when chemicals (usually calcium oxalate and phosphate) that are normally excreted by the kidney become concentrated and are unable to dissolve in solution $(1,2)$. They have become increasingly prevalent over the past two decades with the prevalence rate worldwide being approximately $1.7 \%$ to $8.8 \%$. $5 \%$ of kidney stones are non-calcified with the other $95 \%$ being calcified to varying degrees (3).

Computed Tomography (CT) is the best imaging study to confirm the diagnosis of a urinary stone, and is now the first line imaging study for suspected kidney stone in the United States, however the disadvantages to Computed Tomography involves it being costly and that it uses ionizing radiation (4). In Jamaica, the frontline modalities in patient diagnosis are plain x-ray, computed tomography and ultrasonography. In 2012, there were 28 radiology departments in the island. Plain x-ray was the most widely available modality followed by Computed Tomography and then ultrasonography (5). Non-calcified stones however are usually more difficult to diagnose on radiographs (6).

Except for ultrasonography, radiological scans most commonly used for the diagnosis of kidney stones (these include plain x-ray, CT and fluoroscopy) employs the use of ionizing radiation. This therefore means that patients undergoing these examinations are at a risk for possible radiation health effects which could be either of a Stochastic or Non-Stochastic nature (7).

To protect patients undergoing radiological studies, the International Commission on Radiological Protection (ICRP) has established recommended dose limits for different radiological studies including for those which are used in kidney stone diagnosis such as X-ray of the abdomen, CT scan of the abdomen and intravenous pyelogram. 
This study was therefore aimed at investigating the radiation dose received by patients that undergo these examinations at the University Hospital of the West Indies and compared it with the recommended dose levels of the ICRP as a part of a quality control exercise $(8,9)$.

\section{METHOD}

This study included the use of Thermo-Luminescence Dosimeters (TLD), a PTW Farmer ionization chamber and a 1D water phantom tank. The field of view (FOV) for plain x-ray scan for abdominal studies was determined as shown in fig 1 and the radiation dose within this FOV was measured. The study included the mapping of radiation dose distribution across the surface of the abdomen using TLD films and the measurement of radiation dose fall off with depth as $\mathrm{x}$-ray traverse the abdomen using a water phantom.

The mapping of radiation dose distribution across the surface of the abdomen for abdominal studies involved the use of sixteen TLD films placed across a horizontal surface within the dimensions of $35.56 \times 43.15 \mathrm{~cm}$. This configuration resembles the widest radiation field used by Radiographers to carry out an abdominal x-ray scan. The human abdomen is curved with the greatest distance between the abdomen and back located in the mid sagittal plane. The study however was carried out on a flat surface as a quality control test to investigate the homogeneity of the radiation field as it is distributed across the anatomical surface. The TLDs were paired and separated by a $2 \mathrm{~cm}$ distance at each selected point for measurement within the exposed field (figure 1). 


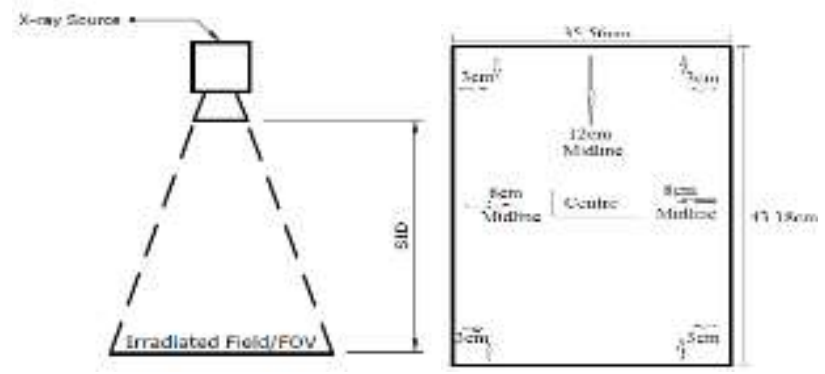

Fig. 1: TLD placement in FOV.

The source - image receptor distance (SID) was set at $101.6 \mathrm{~cm}$ as is used for supine abdominal studies. The source - object distance (SOD) was established at $63.5 \mathrm{~cm}, 50.8 \mathrm{~cm}$ and $25.4 \mathrm{~cm}$ for simulating the SOD of the different body habitus of hypo-sthenic, sthenic and hyper-sthenic respectively. Three sets of exposures were delivered to three different sets of TLD films. The three different set of exposures were set to simulate low, medium and high exposure factors in an effort to analyse the dose distribution across the skin for varying exposure settings, as well as to analyse the tube performance. The TLDs were sent to the International Centre for Environmental and Nuclear Sciences (ICENS) at the University of the West Indies where they were analysed using an automated HARSHAW Model 6600+ reader. This model comes with a built in ${ }^{90} \mathrm{Sr}$ irradiator for calibration purposes.

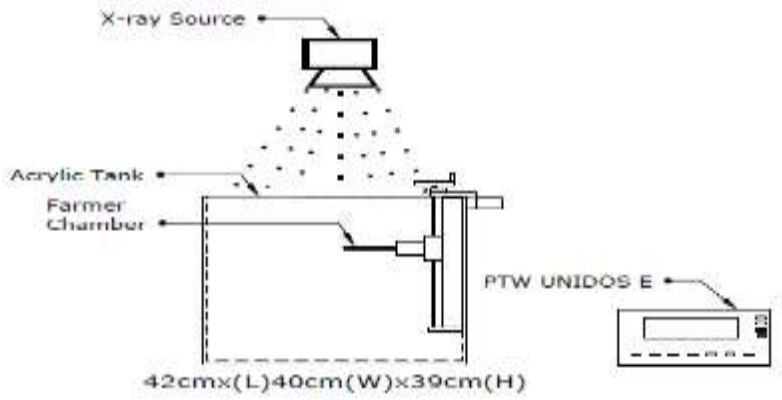

Fig 2. Water Phantom

The measurement of radiation dose fall off with depth as x-ray traverse the abdomen involved the use of a 1D water phantom tank, a $0.6 \mathrm{~cm}^{3}$ PTW Farmer ionization chamber and a PTW 
Unidos E universal dosimeter. The phantom was filled with water to the dimensions of $42 \mathrm{~cm}$ $\mathrm{x} 40 \mathrm{~cm} \times 39 \mathrm{~cm}$ (length, width and height respectively) and placed on the radiographic table and left to rest for 10 minutes. The x-ray tube was then centred to the ion chamber and an SID of $101.6 \mathrm{~cm}$ was used in relation to the table bucky. Two sets of eight exposures were made. Each set of exposure maintained the exposure factors used $(\mathrm{mA}, \mathrm{kV}, \mathrm{mAs})$ and varied the depth of submersion of the ionization chamber. The two set of exposures used simulated the exposure factors normally given to patients of the Sthenic and Hyper-Sthenic body habitus (70kV@20mAs (Parameter 1) and 74kV@40mAs (Parameter 2) respectively). The depth of submersion of the ionization chamber was decreased by $2 \mathrm{~cm}$ for each exposure and ranged from the surface (0) to $14 \mathrm{~cm}$ deep in the water. The data was recorded and analysed.

In assessing the dose received by patients that undergo abdominal CT scans for the diagnosis of kidney stones, the Dose Length Product (DLP) over 3 months for such studies was recorded. Calculations were done to convert the DLP to effective dose using $\mathrm{k}$ factor values from the International Commission on Radiological Protection (ICRP) report 103 (10).

\section{RESULTS}

Figure 3 shows the x-ray surface dose distribution maps for low, medium and high radiographic exposures. The maps were developed using Wolfram Mathematica.

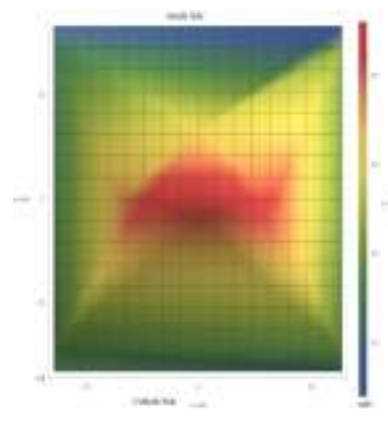

A

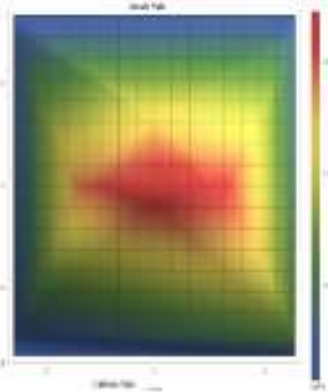

B

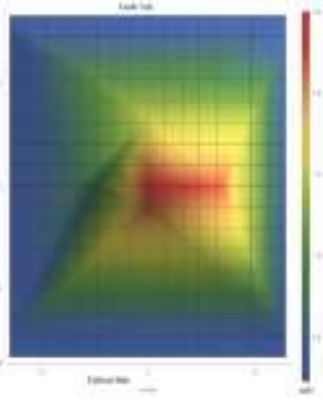

$\mathrm{C}$

Fig. 3: X-ray surface dose distribution for low (A), medium (B) and High (C) exposure. 
For surface dose distribution, the maximum dose was recorded at the centre of the radiation field for all exposures. The dose fell off significantly towards the left of the radiation field in comparison to the right as well as toward the anode side (top) more significantly than towards the cathode side (bottom) for images $\mathrm{A}$ and $\mathrm{B}$ but relatively even for image C. For dose distribution with increased depth, the dose decreased linearly, starting with surface dose readings of $5.45 \mathrm{mGy}$ for parameter $2(74 \mathrm{kV} @ 40 \mathrm{mAs})$ and $2.38 \mathrm{mGy}$ for parameter 1 (70kV@20mAs). The Dose Length Product (DLP) recorded by the CT unit for abdominal scans ranged between $448.1 \mathrm{mGy}^{*} \mathrm{~cm}$ and $1618.1 \mathrm{mGy}^{*} \mathrm{~cm}$ with a mean DLP $940.9 \mathrm{mGy}^{*} \mathrm{~cm}$.

\section{DISCUSSION}

The non-homogenous radiation dose distribution (Figure 3) often times leads to a decrease in the diagnostic quality of radiographs. The persistent sharp fall-off in radiation dose towards the left indicates that there was a misalignment with the collimator light field and the $\mathrm{x}$-ray field (7). There was also evidence of anode heel effect (Figures 3 images A\&B). The anode heel effect stipulates that the x-rays emitted toward the anode side of the tube will be of less energy and intensity than those emitted towards the cathode side of the tube as some of the x-ray photons emitted towards the anode side will pass through the heel of the anode and as such are attenuated to varying extent $(7,11)$.

Over time, depending on the work load of the tube, Pitting may occur where the anode becomes damaged due to constant bombardment from electrons. This increases the heat at the anode surface and results in melting and cracking in the anode (7).The highest equivalent dose for each of the test carried out for plain x-ray scans was recorded at the centre of the radiation field. This is because the distance from the $\mathrm{x}$-ray tube to the dose receptors was shortest at the 
centre as the tube was at a $90^{\circ}$ angle to the centre of the radiation field. Photons therefore not impacting at the centre of the field will therefore travel a longer distance to the dose receptors.

The intensity of radiation photon is inversely proportional to the square of the distance $(7,11)$. Increase distance also means more energy loss due to air kerma (7). Water was used as the tissue simulating material as it has the same effective atomic number as soft tissue (7.4), similar density $\left(1 \mathrm{~g} / \mathrm{cm}^{3}\right.$ for water and $1.05 \mathrm{~g} / \mathrm{cm}^{3}$ for soft tissue) and similar electron density as soft tissue $\left(3.34 \times 10^{26}\right.$ electrons $/ \mathrm{kg}$ for water and $3.36 \times 10^{26}$ electrons $/ \mathrm{kg}$ for soft tissue) (12).

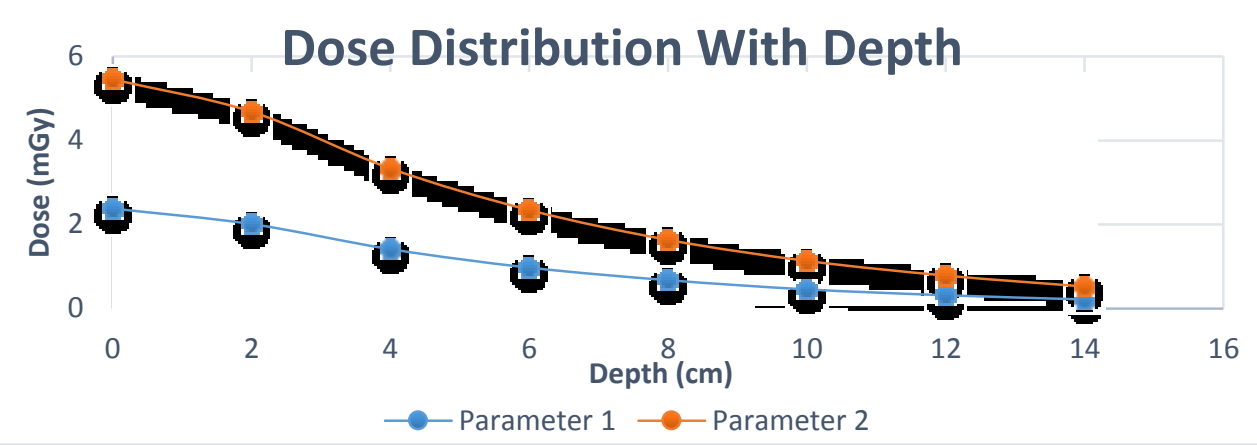

Figure 4: Absorbed dose with depth.

The data in figure 4 shows that for plain x-ray scans a hypersthenic patient will receive a higher skin dose than a sthenic patient; $5.45 \mathrm{mGy}$ and $2.38 \mathrm{mGy}$ respectively. With the weighting factor of $\mathrm{x}$-ray being 1 , this results in a dose equivalent of $5.45 \mathrm{mSv}$ and $2.38 \mathrm{mSv}$ respectively. 


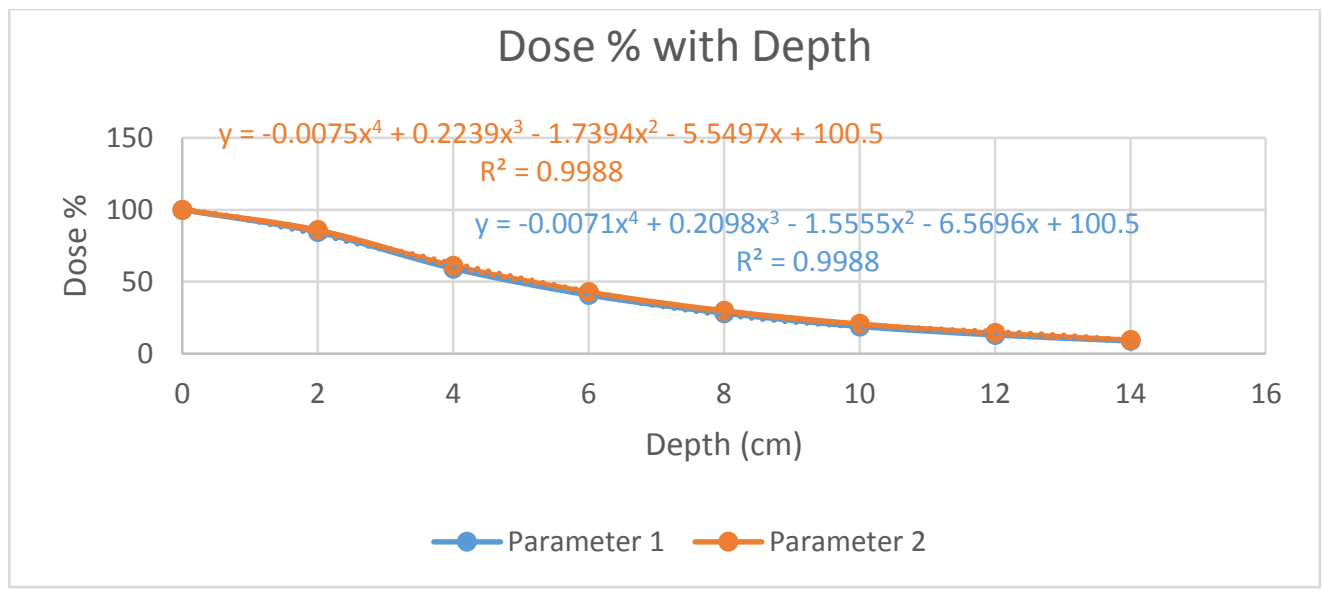

Fig. 5: Percentage depth dose.

Figure 5 shows the dose distribution with depth when both readings have been normalised to $100 \%$. The data has been fitted with the $4^{\text {th }}$ order of polynomial. With $\mathrm{R}^{2}$ values of 0.99 , the data has a high correlation coefficient. The data trajectory suggests a similar behaviour of $\mathrm{x}-$ ray photon interaction with soft tissue for both parameters with parameter 2 having a slightly higher percentage depth dose. This is because as the energy of the photon increases, so too does the percentage depth dose (13).

The table shows the effective dose received by patients that underwent CT abdominal scans over a three-month period. It shows the range of dose length (DLP) product recorded by the unit as well as the mean DLP over this time period.

Table: Effective dose received by patients that underwent CT Abdominal scan.

\begin{tabular}{lll}
\hline & $\begin{array}{l}\text { DLP Range for Abdominal scans } \\
\text { (without contrast) }\left(\mathbf{m G} \mathbf{y}^{*} \mathbf{c m}\right)\end{array}$ & $\begin{array}{l}\text { Mean DLP } \\
\mathbf{( m G \mathbf { H } ^ { * } \mathbf { c m } )} \\
948.1-1618.1\end{array}$ \\
\hline $\begin{array}{l}\text { Effective dose (DLP x } 0.015 \mathrm{mSv} \\
\mathrm{mGy}-1 \mathrm{~cm}-1)\end{array}$ & $6.72 \mathrm{mSv}-24.27 \mathrm{mSv}$ & $14.11 \mathrm{mSv}$ \\
\hline
\end{tabular}


Calculations were done to convert the DLP to effective dose using $\mathrm{k}$ factor values from ICRP 103 (DLP x k factor for abdomen) (10). The dose readings for CT scans varied depending on the slice thickness and patient habitus. A decrease slice thickness results in an increase in image noise and as such an increase in mAs is necessary to combat the increase in image noise (14).

For scans of the abdomen for renal calculi, the entire abdomen is exposed to radiation. This leads to irradiation of all organs in the abdominal and pelvic cavity. Some organs are more radiosensitive than others and are given weighting factor numbers $\left(\mathrm{W}_{\mathrm{T}}\right)$. The higher the tissue weighting factor number, the more radiosensitive the organ is (15). There is no specific value for the tissue weighting factor for kidney, however other organs that are often exposed during an X-ray or CT scan for kidney stones and are more radiosensitive includes the Bone Marrow, Stomach and Colon with a $\mathrm{W}_{\mathrm{T}}$ of 0.12 and the Gonads with a $\mathrm{W}_{\mathrm{T}}$ of 0.08 . Moderately radiosensitive tissues such as the Bladder and Liver $\left(0.04 \mathrm{~W}_{\mathrm{T}}\right)$ are also irradiated (15).

The irradiation of these tissues increases the risk of stochastic effects to these organs more than to the kidneys and as such the use of ionizing radiation scans should be employed with caution. Despite the non-homogenous distribution of radiation dose in the irradiated field, all recorded dose conformed to those estimated by the ICRP for abdominal and urinary studies $(8,9)$.

\section{CONCLUSION}

Skin dose is greatest at the centre of the abdomen for plain x-ray abdominal scans. Radiation equivalent dose to the skin ranged from $2.38 \mathrm{mSv}$ for a sthenic body habitus patient to $5.45 \mathrm{mSv}$ for a hypersthenic patient. For CT scans, effective dose was found to range between $6.72 \mathrm{mSv}$ and $24.27 \mathrm{mSv}$. All radiation dose measured were found to be within recommended ICRP 
operational limits $(8,9)$. The developed procedure could be implemented in other radiology departments in Jamaica as a part of their quality assurance program.

\section{ACKNOWLEDGEMENT}

We thank the International Atomic Energy Agency (IAEA) for fellowship training and equipment supplied through technical corporation programme. We also thank Mr Clifton McCook for his assistance in coding the Wolfram Mathematica software. 


\section{REFERENCES}

1. Morrison B. Hot days, increased risk of kidney stones Jamaica: The Jamaica Observer; 2014 [cited 2016 May 9]. Available from: http://www.jamaicaobserver.com/news/Hotdays--increased-risk-of-kidney-stones_17195585

2. Jamaica: Jamaica Urological Society [cited 2016 May 9, 2016]. Available from: http://www.jus.org.jm/faq.php

3. Chang-Xu CZ, Wang XL, Liu TZ, Zeng X-T, Li S, Duan X-W. Self-fluid management in Prevention of Kidney Stones: A PRISMA-Compliant Systematic Review and DoseResponse Meta-Analysis of Observational Studies. Medicine 201594.

4. Moore C, Daniels B, Ghita M, Gunabushanam G, Luty S, Molinaro A et al. Accuracy of reduced-dose computed tomography for ureteral stones in emergency department patients, 2014.

5. List of Health Facilities Non-Governmental organizations/community based organization and International Agencies In: Health Mo, editor. Jamaica 2012.

6. Kowalczyk N, Mace J. Radiographic pathology for technologists: Mosby Elsevier; 2009.

7. Bushong SC. Radiologic science for technologists: physics, biology, and protection. 10 ed. United States of America Elsevier Mosby; 2013.

8. What are the Radiation Risks from CT? United States of America United States Food and Drug Administration [cited 2016 May 9]. Available from: http://www.fda.gov/RadiationEmittingProducts/RadiationEmittingProductsandProcedures/MedicalImaging/Medical X-Rays/ucm115329.htm

9. Radiation Dose in X-Ray and CT Exams [cited 2016 May 9]. Available from: http://www.radiologyinfo.org/en/info.cfm?pg=safety-xray 
10. AAPM. The measurement, reporting, and management of radiation dose in CT. United States of America American Association of Physicists in Medicine 200896.

11. Fauber T. Radiographic imaging and exposure 3ed. United States of America: Mosby Elsevier; 2009.

12. Hasegawa B. Physics of Medical X-ray Imaging. 2 ed. United States of America Medical Physics Publishing Corporation 01 Sep 1991

13. Gazda M, Coia L. Principles of Radiation Therapy. In: Cancer Management: A Multidisciplinary Approach [Internet]. Available from: http://www.thymic.org/uploads/reference_sub/02radtherapy.pdf

14. Goldman L. Principles of CT: radiation dose and image quality. Society of Nuclear Medicine 2007.

15. Health effects of security scanners for passenger screening (based on X-ray technology). Europe: Scientific Committee on Emerging and Newly Identified Health Risks (SCENIHR) 2012 April 26. Report No. 